\section{A positive feedback loop couples Ras activation and CD44 alternative splicing}

\author{
Chonghui Cheng, ${ }^{1}$ Michael B. Yaffe, ${ }^{1,2}$ and \\ Phillip A. Sharp ${ }^{1,2,3}$ \\ ${ }^{1}$ Center for Cancer Research, ${ }^{2}$ Department of Biology, \\ Massachusetts Institute of Technology, \\ Cambridge, Massachusetts 02139, USA
}

The Ras signaling pathway is important in both cell proliferation and tumor progression. Alternatively spliced isoforms of CD44 containing variable exon 6 (v6) can serve as coreceptors for growth factor receptors that activate Ras. Here we use v6-specific small interfering RNA (siRNA) to investigate the role of CD44 alternative splicing in Ras signaling. We identify a positive feedback loop in which Ras signaling promotes CD44v6 splicing, and CD44v6 then sustains late Ras signaling, which is important for cell cycle progression. These results are the first demonstration of a positive feedback loop linking signaling-dependent alternative splicing to mitogenic progression.

Supplemental material is available at http://www.genesdev.org.

Received March 20, 2006; revised version accepted April 27, 2006.

The transmembrane glycoprotein CD44 has been implicated in cell adhesion, migration, invasion, and proliferation (for a recent review, see Ponta et al. 2003). This diversity of functions may reflect the activities of its multiple alternatively spliced isoforms. CD44 contains nine or 10 variable exons residing between constitutive exons 5 and 6 . Peptides encoded by these variable exons are located in the extracellular domain of CD44 and are thought to modulate the cellular response to growth factor stimulation (Bennett et al. 1995; Sherman et al. 1998; van der Voort et al. 1999; Orian-Rousseau et al. 2002; Yu et al. 2002; Ponta et al. 2003). The standard form of CD44, which excludes all variable exons, is expressed in the majority of vertebrate tissues. CD44 isoforms or variants, which include different combinations of the variable exons, are mainly expressed in proliferating cells and tumors. The expression levels of these isoforms correlate with enhanced malignancy and invasiveness (Gunthert et al. 1991; Muller et al. 1997; Martin et al. 2003; Sonobe et al. 2005).

The production of CD44 variants through alternative splicing is regulated by splicing factors, such as Sam68 and SRm160, and stimulated by Ras/MAPK (mitogenactivated protein kinase) signaling (Ras-Raf-MEK-ERK)

[Keywords: Ras; CD44 variants; alternative splicing; cell cycle] ${ }^{3}$ Corresponding author

E-MAIL sharppa@mit.edu; FAX (617) 253-3867.

Article is online at http://www.genesdev.org/cgi/doi/10.1101/gad.1430906.
(Weg-Remers et al. 2001; Matter et al. 2002; Cheng and Sharp 2006). These splicing factors depend upon exon splicing enhancers in the CD44 variable exons. Their activity in promoting the inclusion of CD44 variable exons is controlled by Ras/MAPK signaling, at least in part through modification of splicing factors at the level of phosphorylation. However, the signaling pathway between Ras/MAPK activation and stimulation of alternative splicing in the nucleus is not well defined.

Interestingly, CD44 variants containing variable exon 6 (v6) have been shown to be important for Ras activation through formation of a coreceptor complex with the hepatocyte growth factor HGF and its receptor tyrosine kinase Met, whose phosphorylation activates Ras signaling (Orian-Rousseau et al. 2002). These observations led us to postulate that Ras/MAPK activation and CD44 variant expression might be coordinately regulated through a positive feedback loop (Fig. 1).

Addition of growth factors to quiescent cells results in biphasic activation of the Ras/PI3k/MAPK signaling pathways (Cook and McCormick 1996; Grammer and Blenis 1997; Weber et al. 1997; Jones and Kazlauskas 2001a; Murphy et al. 2002), with an early rapid increase in MAPK activation from 5 to $60 \mathrm{~min}$, followed by a late second wave of MAPK activation of lower amplitude beginning at $\sim 7-10 \mathrm{~h}$. This second wave of MAPK activity is sustained for hours after growth factor stimulation and is critical for cells to progress from G1 into S phase (Cook and McCormick 1996; Weber et al. 1997; Jones and Kazlauskas 2001a,b). Despite its importance, however, mechanisms underlying the sustained activity of Ras/MAPK signaling have remained elusive. Here we demonstrate a positive feedback loop that couples sustained Ras/MAPK signaling to CD44 alternative splicing. Ras/MAPK activation is both necessary and sufficient to stimulate alternative CD44 splicing. In conjunction with growth factors and their receptor tyrosine kinases, specific CD44v6 variants produced by alternative splicing subsequently enhance the late wave of Ras/ MAPK activation to facilitate cell cycle progression.

\section{Results and Discussion}

\section{CD44 variants are up-regulated upon serum stimulation}

To investigate whether growth factor stimulation upregulates CD44 variants, serum factor-depleted HeLa cells were stimulated by addition of serum-rich media. The levels of CD44 variants were monitored by quantitative RT-PCR using different sets of primers (Fig. 2A). A set of variants were detected using a $5^{\prime}$ primer from a constitutive exon and two different 3 ' primers complementary to either v6 or v8 exon, respectively. In addition, the CD44 standard form was detected using primers that base-pair to the constitutive exons. The v6 primer and standard primer predominantly amplified a single product (Fig. 2A). The v8 primer gave rise to three spliced variants containing (1) variant exons v6, v7, and v8 (referred as v6-8); (2) variant exons v3 and v8 (v3.8); and (3) variant exon v8 (v8), all joined to the 5 ' constitutive exon (Fig. 2A, all products were confirmed by DNA sequencing). Following $24 \mathrm{~h}$ of serum starvation, 


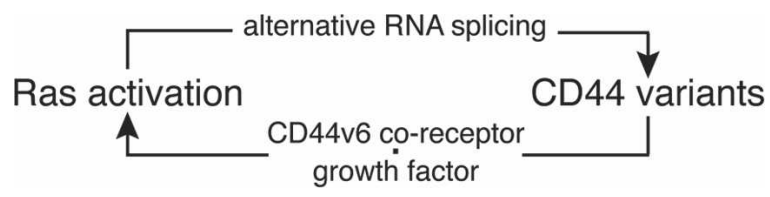

Figure 1. A model of a positive feedback loop between Ras activation and CD44 variants. Activated Ras signaling stimulates CD44 alternative splicing, resulting in the production of CD44 variants. Subsequently, the spliced CD44 variants containing v6 exon act as coreceptors. They form complexes with growth factors and their receptor tyrosine kinases and further activate Ras signaling.

the relative expression levels of CD44 variants were low. Stimulation of these cells with serum resulted in upregulation of v6 expression (Fig. 2B). This stimulation was apparent after $2 \mathrm{~h}$, peaked at $\sim 8 \mathrm{~h}(6.75 \pm 1.67$-fold $)$, and decreased to basal level at $\sim 24-32 \mathrm{~h}$ due to the depletion of serum factors. Expression of the v8 variants and other examined variants (v3 and v10) showed a similar time course of up-regulation (Fig. 2B; data not shown). In contrast, expression of the CD44 standard form showed less variation upon serum starvation and stimulation. An approximately twofold $(1.74 \pm 0.68)$ increase was observed after $8 \mathrm{~h}$ of stimulation as compared with the unstimulated state. The expression levels of CD44 variants were also examined using $5^{\prime}$ primers complementary to individual variable exons and a primer to the $3^{\prime}$ constitutive exon. Similar results were obtained with these primer sets (data not shown). Overall, these results indicate that CD44 alternative splicing is up-regulated by serum.

\section{Expression of CD44 variants is dependent on $v 6$ isoforms}

If alternative splicing of CD44 and Ras/MAPK signaling were components of a positive feedback loop (Fig. 1), then expression of CD44 variants should depend on CD44v6 isoforms, since these are known to be important for growth factor-dependent activation of Ras/MAPK (Orian-Rousseau et al. 2002). Thus, down-regulation of the v6-containing isoforms would be expected to result in disruption of the positive feedback loop, with subsequent reduction of other CD44 isoforms, which do not contain v6. To test this hypothesis, we used v6 exonspecific small interfering RNA (siRNA) that only knocks down v6-containing isoforms. v6 siRNA transfection resulted in an approximately sevenfold knockdown (14 $\pm 4.5 \%$ expression levels of control cells) of mRNAs containing this exon compared with a control siRNA with no complementarity to any known human sequence (see Fig. 3A, v6 and v6-8 PCR products). Flow cytometry analysis using a v6-specific antibody also showed threefold or greater knockdown in protein level (data not shown). As predicted by the existence of a positive feedback loop, this down-regulation of $\mathrm{v} 6$ isoform resulted in a reduction in the expression of many other CD44 variants that do not contain the v6 exon, in response to serum stimulation (Fig. 3A left panel; Supplementary Fig. S1 for analyses of isoforms containing v8, v9, and v10). For example, the levels of v3.8 and v8 variants were reduced to levels comparable to that of the v6-containing variant v6-8 $(27 \pm 11 \%$ and $23 \pm 2.9 \%$ of control siRNA-treated cells, respectively). This v6 siRNA effect is specific to mRNA-containing CD44 vari- ants as the level of standard form was not significantly altered $(87 \pm 4.6 \%)$. Similar results were obtained using a second v6 siRNA (data not shown).

Since CD44 alternative splicing is known to be regulated by Ras/MAPK signaling and since CD44v6 variants, in particular, enhance growth factor-dependent activation of the Ras/MAPK signaling cascade, we next examined whether down-regulation of CD44v6 impaired Ras/MAPK signaling. Since cell surface expression of CD44 variants involves synthesis of mRNAs and translation of CD44 variants, we focused on levels of Erk phosphorylation at $8 \mathrm{~h}$ after serum stimulation. This correlated with the maximum expression levels of CD44v6 (Fig. 2B). As shown in Figure 3B, when v6 siRNA-treated cells were stimulated with serum, they displayed a dramatic decrease in the extent of Erk phosphorylation $8 \mathrm{~h}$ later compared with control siRNAtreated cells. The amount of Erk protein, however, was not altered.

The studies in Figure 3B show that alternative splicing of CD44 to generate the v6 isoforms is important for activation of the Ras/MAPK pathway by serum. If CD44v6 splicing and Ras/MAPK activation were part of a feed-forward loop, then conversely, constitutive activation of Ras should eliminate the requirement of v6containing complexes for further production of CD44 variants. Accordingly, v6 siRNA treatment should no longer decrease the inclusion of the non-v6-containing variants. To test this, a constitutively activated mutant

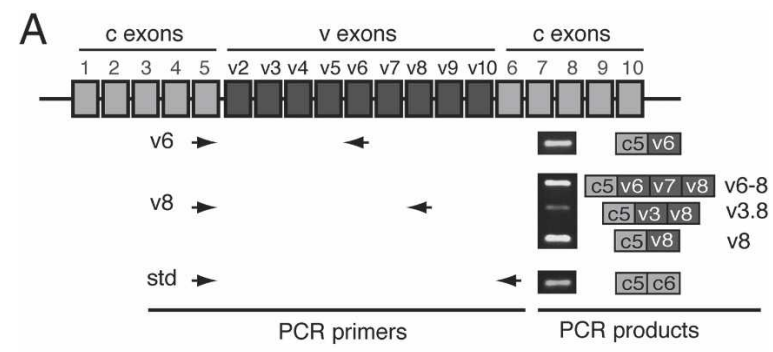

B

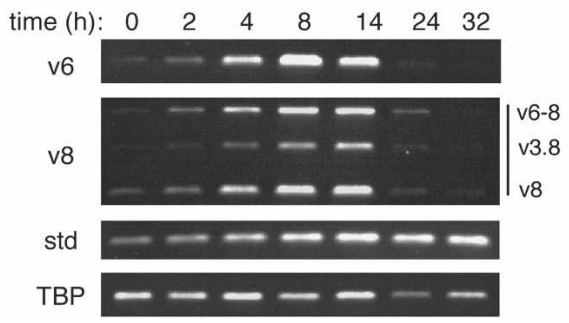

Figure 2. Expression of CD44 variants is stimulated by growth factors in serum. (A) A schematic diagram of the CD44 gene. Both constitutive (c) and variable (v) exons are depicted. The PCR primers used to amplify CD44 variable and standard (std) isoforms are shown as arrowheads. The primers for both the $\mathrm{v} 6$ and standard isoforms predominantly generate one PCR product, whereas the primers for the v8 variants amplify three splice variants. Structures of the PCR products are depicted to the right of the individual example gels. $(B)$ A time course of serum stimulation. Serum-starved HeLa cells were stimulated with $10 \%$ serum and collected at the indicated time points. RNA was extracted, and quantitative RT-PCR was performed. Agarose gels of the PCR products are shown. The numbers of PCR cycles and exposure length of the images for each set of PCR primers vary and cannot be directly compared. PCR primers used in each reaction are depicted to the left of the gels. TATA-binding protein (TBP) served as a loading control. 

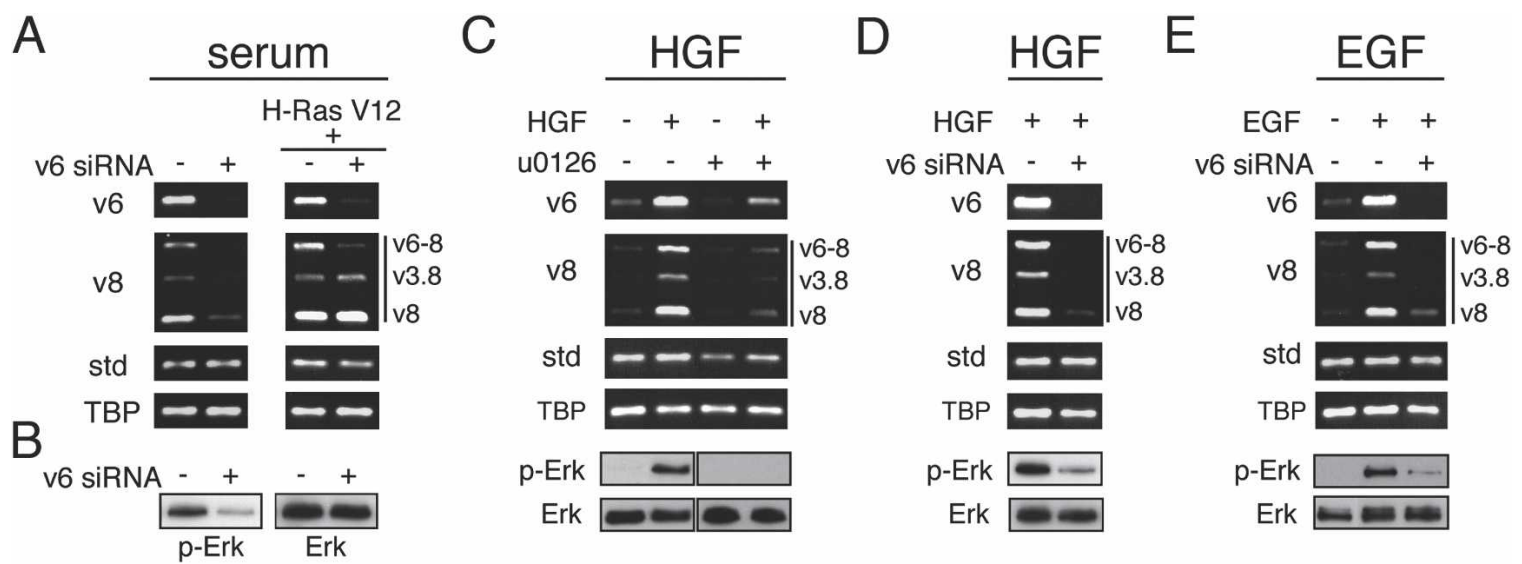

Figure 3. siRNA-mediated knockdown of CD44v6 affects alternative splicing of CD44 and down-regulates Erk activity. (A) RT-PCR analyses of CD44 variants in HeLa cells transfected with control siRNA or v6 siRNA followed by serum stimulation for 8 h (left panel), or transfected with an additional H-Ras V12 construct (right panel). TBP was used as a loading control. (B) Western blot analyses of Erk phosphorylation following serum stimulation for $8 \mathrm{~h}$ in cells transfected with control or v6 siRNA. Antibodies recognizing phosphorylated Erk (p-Erk) or total Erk (Erk) were used. (C-E) RT-PCR analyses of CD44 variants and Western blot analyses of Erk phosphorylation in serum-starved HeLa cells that were stimulated with HGF $(60 \mathrm{ng} / \mathrm{mL})$ for $8 \mathrm{~h}$ in the absence or presence of U0126 $(25 \mu \mathrm{M})(C)$, or in serum starved control or v6 siRNA-treated HeLa that were stimulated with $\operatorname{HGF}(D)$ or EGF $(5 \mathrm{ng} / \mathrm{mL}, E)$ for $8 \mathrm{~h}$.

H-Ras V12 construct was cotransfected along with v6 siRNA. As shown in Figure 3A, right panel, after ectopically expressing H-Ras V12, isoforms containing v6 were similarly silenced by v6 siRNA treatment ( $16 \pm 5.8 \%$ of control levels) as compared with control cells. However, the levels of the other two variants (v3.8 and v8) were almost identical after treatment with either v6 siRNA or control siRNA in cells expressing H-Ras V12 $(84 \pm 7.0 \%$ and $99 \pm 7.7 \%$, respectively, to the levels of control siRNA-treated cells). As an internal control, the v6-8 variant amplified in the same PCR reaction maintained down-regulated levels $(20 \pm 6.0 \%$ of control levels) because this variant contained the $\mathrm{v} 6$ exon targeted by v6 siRNA. These findings provide strong support for a positive feedback loop in which specific isoform-dependent activation of Ras/MAPK signaling in response to serum results in additional synthesis of these CD44 isoforms.

\section{The growth factors HGF and EGF (epithelial growth factor) activate Ras/MAPK signaling dependent on CD44v6}

To investigate whether CD44v6 variants are important for activation of Ras/MAPK signaling in response to specific growth factors, we examined the effects of HGF and EGF on these cells. Previous studies have shown that HGF stimulates Ras activation in a CD44-dependent manner (Orian-Rousseau et al. 2002). We therefore measured the levels of CD44 variants after HGF stimulation by RT-PCR. As shown in Figure 3C, at $8 \mathrm{~h}$ following HGF addition, the expression of variants containing the v6 exon was increased by approximately fourfold $(4.2 \pm 0.67)$. A similar increase was observed for the v6-8, v3.8, and v8 variants $(4.9 \pm 1.5,5.6 \pm 1.2$, and $5.0 \pm 0.85$ fold, respectively). In contrast, the standard form was only slightly increased (1.35 \pm 0.33 -fold). Addition of an inhibitor of MEK/Erk, U0126 (Favata et al. 1998), to HGF-treated cells inhibited the up-regulation of all of examined CD44 variants, indicating that Ras/MAPK signaling is required for HGF-induced alternative splicing of CD44. The residual expression of CD44 variants after U0126 treatment suggests that other signaling cascades, in addition to Ras/MAPK, may also regulate alternative splicing of CD44. Similar to what we observed with serum stimulation, down-regulation of v6 isoforms using siRNA impaired the ability of HGF to activate Erk at $8 \mathrm{~h}$, as well as to stimulate Ras/MAPK-dependent alternative splicing of CD44 (Fig. 3D). These results further support a positive feedback loop between CD44 variants and Ras activation, and suggest that $\mathrm{v} 6$ isoforms are required for Ras/MAPK-dependent CD44 alternative splicing in response to HGF.

The observations that CD44v6 is important for both serum- and HGF-dependent Ras/MAPK activity suggested that these CD44 variants might be important for signaling events downstream of additional receptor tyrosine kinases. Since CD44 has been previously shown to interact with members of the epidermal growth factor receptor (EGFR) family (Bourguignon et al. 1997; Sherman et al. 2000; Yu et al. 2002), we tested whether EGF could also stimulate the alternative splicing of CD44 and whether this stimulation was dependent on v6 isoforms. Cells were transfected with either control siRNA or v6 siRNA and stimulated with EGF for $8 \mathrm{~h}$. In control siRNA-treated cells, expression of CD44 variants was stimulated approximately fourfold by EGF (Fig. 3E). This induction was similar to that observed in cells treated with EGF in the absence of siRNA (data not shown). In contrast, EGF-dependent up-regulation of CD44 variants was almost completely inhibited in the v6 siRNAtreated cells (v3.8, sixfold; v8, 4.3-fold reduction). Similar to what we observed with serum and HGF, downregulation of v6-containing CD44 isoforms significantly impaired the activation of Erk at $8 \mathrm{~h}$ following EGF addition (Fig. 3E), suggesting that CD44 variants may act as coreceptors for different growth factors. Taken together, these results indicate a positive feedback loop coupling Ras/MAPK signaling and CD44 alternative splicing. In this loop, Ras/MAPK signaling stimulates CD44 alternative splicing through the regulation of splicing factors. Alternative splicing of the CD44 gene generates CD44 
variants. The v6 exon-containing variants then form coreceptor complexes with growth factor/receptor tyrosine kinase, which in turn sustains Ras/MAPK signaling. The mechanisms underlying the dependence of sustained Ras/MAPK activation by different growth factors on the expression of the CD44v6 isoform are not clear. Previous studies have shown that the CD44v6 isoform can complex with the Met receptor for HGF signaling. This could also be the case for other receptors. Alternatively, CD44v6 might antagonize inhibitors, such as protein phosphatases, to prolong the activity of growth factordependent signaling. In addition, CD44v6 could sustain cellular signaling through an autocrine loop, where this isoform is conjectured to be important for a secreted factor. In fact, sustained EGFR signaling frequently involves an autocrine loop.

\section{Knockdown of CD44v6 inhibits sustained Erk activation and impairs G1-S transition}

Positive feedback loops altering Ras/MAKP signaling could lead to sustained changes in cellular properties. For example, the positive feedback loop coupling CD44 alternative splicing and Ras activation presented here could produce sustained Ras signaling, which is important for cell cycle progression. Previous studies have shown that overexpression of a CD44v6 variant up-regulates Ras/MAPK activation and promotes cell proliferation (Marhaba et al. 2005). To investigate the role of the positive feedback loop in cell cycle progression, we examined the time course of Erk activation, and the progression of quiescent cells into $S$ phase in cells treated with v6 siRNA. As shown in Figure 4A, both control and v6 siRNA-treated cells showed robust and equivalent activation of the Ras/MAPK pathway at early times after EGF addition (5-60 min). Control cells also displayed a sustained pattern of low amplitude Erk activation between 4 and $12 \mathrm{~h}$ (last time point taken). This late wave of Erk activity was dramatically impaired in cells treated with CD44v6 siRNA.

Late Ras/MAPK signaling after growth factor stimulation is required for cells to transit from G0/G1 into S phase (Cook and McCormick 1996; Weber et al. 1997; Jones and Kazlauskas 2001a,b). We therefore analyzed the cell cycle profiles of control or v6 siRNA-treated HeLa cells that were synchronized in G2/M phase using nocodazole, and released by drug removal and serum stimulation. Eight hours after release, $>95 \%$ of cells, treated with either control or CD44v6 siRNA, were in G1. After an additional $2 \mathrm{~h}, \sim 24 \%$ of the control siRNAtreated cells had entered S phase. In contrast, only $12 \%$ of the CD44v6 siRNA-treated cells had progressed into $S$ phase at this time, indicating a delay of S-phase entry following down-regulation of the CD44v6 isoform (Supplementary Fig. S2).

To eliminate any complications arising from nocodazole-induced G2/M arrest, we switched to a glioblastoma cell line, T98G, that arrests in a quiescent G0/G1 phase upon serum withdrawal, in contrast to HeLa cells. As in HeLa cells, T98G cells up-regulated CD44 variants upon serum stimulation. CD44v6 siRNA treatment down-regulated the levels of v6 variants and impaired Erk phosphorylation in the late phase (Supplementary Fig. S3). Quiescent control and CD44v6 siRNA-treated T98G cells were synchronously induced to re-enter the cell cycle by serum refeeding, and cell cycle progression

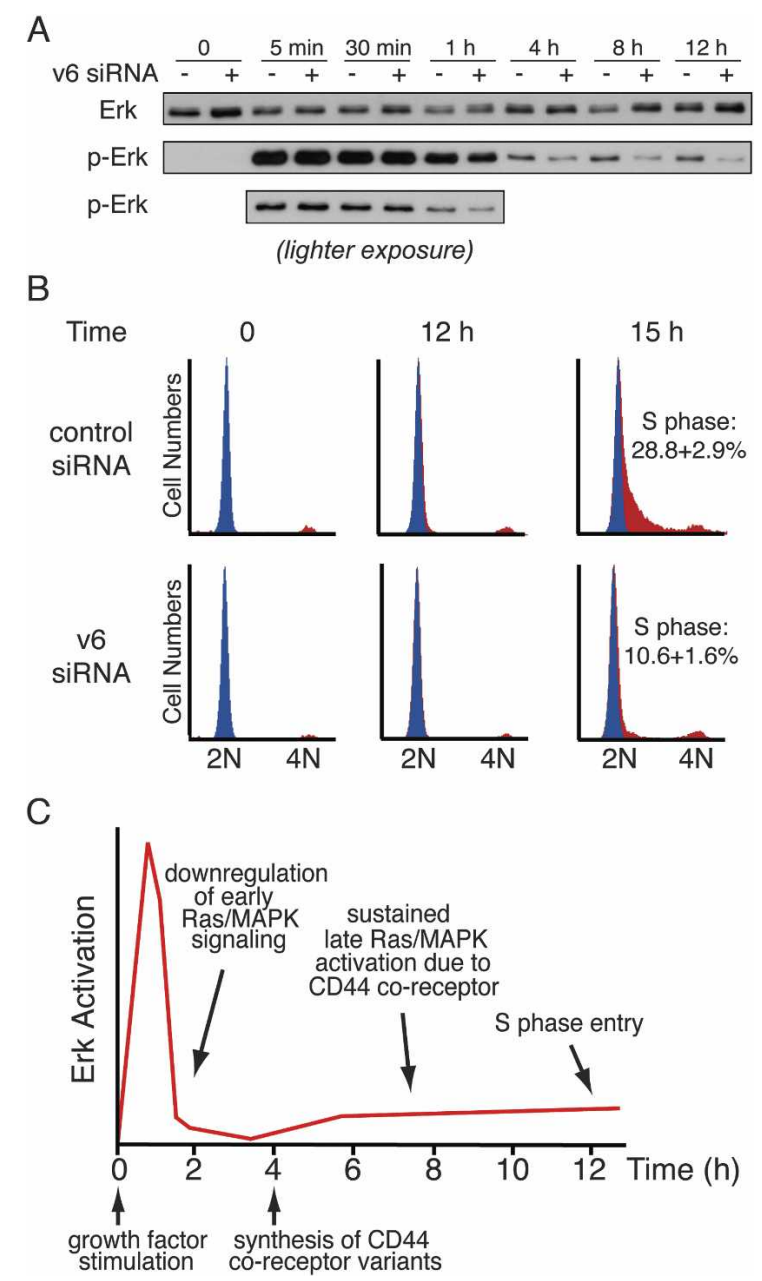

Figure 4. Down-regulation of CD44v6 impairs sustained Erk activation and inhibits the G1-S transition. (A) Western blot analyses of Erk activation in EGF-stimulated HeLa cells transfected with control or CD44v6 siRNA. These cells were collected at indicated times after EGF addition. (B) Cell cycle analyses of serum-starved T98G cells, treated with control or CD44v6 siRNA, and stimulated with serum to re-enter the cell cycle. The blue peak represents cells in G0/G1 phase at time $0 \mathrm{~h}$. This peak is overlaid on the subsequent time points for each experiment. The percentage of cells in S phase at $15 \mathrm{~h}$ after serum stimulation is indicated. (C) A model for sustained Ras/MAPK signaling and G1-S transition, dependent on CD44 coreceptor variants. See text for details.

was analyzed by flow cytometry. As shown in Figure 4B, $12 \mathrm{~h}$ after serum stimulation, the control siRNA-treated cells had begun to progress into S phase $(\sim 8 \%)$. Almost all of the CD44v6 siRNA-treated cells, however, remained in G0/G1 ( 4\%). By $15 \mathrm{~h}$ after stimulation, $\sim 29 \%$ of the control cells were transiting through $S$ phase, compared with only $\sim 11 \%$ of the v6 siRNA-treated cells. These results indicate that positive feedback of CD44v6dependent Ras/MAPK signaling at late times following growth factors stimulation is involved in triggering proper cell cycle progression.

Our data, together with previous findings, suggest a new mechanistic model for the biphasic activation of Ras/MAPK (Fig. 4C). Upon growth factor stimulation, receptor tyrosine kinases generate an early burst of Erk activation independent of CD44v6 variants. This signal is rapidly down-regulated; however, it initiates a positive 
feedback loop by stimulating isoform-specific CD44 expression through alternative splicing. These newly synthesized CD44 coreceptor variants containing v6 exon, in conjunction with growth factors and their receptor tyrosine kinases, then facilitate late Ras/MAPK signaling for a sufficient time to drive cell cycle progression. The positive feedback loop described above may be important for the transition from normal to transformed phenotypes. Maintenance of this positive feedback loop, for example, could constitute a mechanism for persistent Ras activation in tumors that lack oncogenic mutations in the Ras genes (Guha et al. 1997; von Lintig et al. 2000). The use of exon-specific siRNA in our study revealed new insights into how regulated alternative splicing can control intracellular signaling, engage cell proliferation, and potentially influence tumor progression. This study illustrates, for the first time, a mechanism for long-lasting changes in cell state maintained at the level of RNA splicing.

\section{Materials and methods}

\section{Cells and transfection}

Human HeLa and T98G cells were maintained in Dulbecco's modified Eagle's medium (DMEM) (HeLa) or RPMI (T98G), both containing 10\% fetal bovine serum (IFS), penicillin, and streptomycin. RNA interference (RNAi) experiments were performed using Oligofectamine (Invitrogen) and siRNAs (100 pmol) for each well of a six-well plate. Cells were harvested $60-72$ h post-transfection. For H-Ras V12 overexpression experiments, siRNA, H-Ras V12, and a GFP plasmid were cotransfected in HeLa cells using lipofectamine 2000 (Invitrogen). Twenty-four hours after transfection, these cells were trypsinized and sorted by GFP expression. The GFP-positive cells were selected and incubated for an additional $24 \mathrm{~h}$ before collection of cells. siRNA sequences of the sense strands are as follows: CD44 v6-1, GCAACTCCTAGTAGTACAAdTdT; CD44 v6-2, TGAGGGATATCGCCAAACAdTdT. For cell cycle analysis, HeLa cells were synchronized in G2/M phase using $200 \mathrm{ng} / \mathrm{mL}$ Nocodazole for $18 \mathrm{~h}$. T98G cells were synchronized in G0/G1 phase by serum starvation. Cell cycle re-entry was analyzed using flow cytometry with propidium iodid staining at different time points after removing drug (HeLa) and serum stimulation of synchronized cells.

\section{RT-PCR analysis}

RNA was prepared using the Qiagen RNeasy kit. RNA $(0.25 \mu \mathrm{g})$ was used in an RT reaction in a $20-\mu \mathrm{L}$ volume for $1 \mathrm{~h}$. Two microliters of the resulting cDNA were subjected to PCR reactions using specific primers. Each PCR cycle includes $45 \mathrm{sec}$ at $94^{\circ} \mathrm{C}, 45 \mathrm{sec}$ at $55^{\circ} \mathrm{C}$, and $45 \mathrm{sec}$ at $72^{\circ} \mathrm{C}$. Thirty cycles were used for amplification of v6 variants, 29 cycles for v8, 28 cycles for standard CD44, and 31 cycles for TBP. PCR products were analyzed on $1.5 \%$ agarose gels. Quantitative analysis of intensities of PCR products was performed using AlphaImager 2200, V5.5 (Alpha Innotech Corporation). The intensity values were normalized to TBP (loading control) and compared with that of control cells. Error bars represent standard deviation. The primer sequences for amplifying CD44 variants and standard form are as follows: a forward primer complementary to the $5^{\prime}$ constitutive exon (5'-CATCCCAGACGAAGACAGTC-3'); reverse primers base-pairing to specific variable exons v6 $\left(5^{\prime}\right.$-CAGCT GTCCCTGTTGTCGAA-3') and v8 (5'-GTTGTCATTGAAAGAGGTC CT- $\left.3^{\prime}\right)$; and a reverse primer complementary to the $3^{\prime}$ constitutive exon (5'-TTTGCTCCACCTTCTTGACTCC-3').

Western blot analysis

Cell lysates were prepared in RIPA buffer ( $1 \%$ NP40, $0.5 \%$ sodium deoxycholate, $0.1 \%$ SDS, in phosphate buffered saline at $\mathrm{pH} 7.4)$, supplemented with protease inhibitors (complete tablet from Roche) and phosphatase inhibitors ( $2 \mathrm{mM}$ sodium pyrophosphate, $50 \mathrm{mM} \mathrm{NaF}, 5 \mathrm{mM}$ $\beta$-glycerophosphate). Samples were immunoblotted with antibodies to total Erk and the activated phosphorylated form (p-Erk), both from Cell Signaling Technology.

\section{Acknowledgments}

We thank L. Aleman, A. Seila, D. Tantin, M. Wernett, S. Erkeland, A. Grishok, M. Ebert, A. Garfinkel, and J. Neilson for discussion and critical reading of the manuscript, and J. Wang for excellent technical help. This research was supported in part by grants from the National Institute of Health and National Cancer Institute (to P.A.S. and M.B.Y.) and by a post-doctoral fellowship from the Damon Runyon Cancer Research Foundation (to C.C.).

\section{References}

Bennett, K.L., Jackson, D.G., Simon, J.C., Tanczos, E., Peach, R., Modrell, B., Stamenkovic, I., Plowman, G., and Aruffo, A. 1995. CD44 isoforms containing exon V3 are responsible for the presentation of heparin-binding growth factor. J. Cell Biol. 128: 687-698.

Bourguignon, L.Y., Zhu, H., Chu, A., Iida, N., Zhang, L., and Hung, M.C. 1997. Interaction between the adhesion receptor, CD44, and the oncogene product, p185HER2, promotes human ovarian tumor cell activation. J. Biol. Chem. 272: 27913-27918.

Cheng, C. and Sharp, P.A. 2006. Regulation of CD44 alternative splicing by SRm160 and its potential role in tumor cell invasion. Mol. Cell. Biol. 26: 362-370.

Cook, S.J. and McCormick, F. 1996. Kinetic and biochemical correlation between sustained p44ERK1 (44 kDa extracellular signal-regulated kinase 1) activation and lysophosphatidic acid-stimulated DNA synthesis in Rat-1 cells. Biochem. J. 320: 237-245.

Favata, M.F., Horiuchi, K.Y., Manos, E.J., Daulerio, A.J., Stradley, D.A., Feeser, W.S., Van Dyk, D.E., Pitts, W.J., Earl, R.A., Hobbs, F., et al. 1998. Identification of a novel inhibitor of mitogen-activated protein kinase kinase. J. Biol. Chem. 273: 18623-18632.

Grammer, T.C. and Blenis, J. 1997. Evidence for MEK-independent pathways regulating the prolonged activation of the ERK-MAP kinases. Oncogene 14: 1635-1642.

Guha, A., Feldkamp, M.M., Lau, N., Boss, G., and Pawson, A. 1997. Proliferation of human malignant astrocytomas is dependent on Ras activation. Oncogene 15: 2755-2765.

Gunthert, U., Hofmann, M., Rudy, W., Reber, S., Zoller, M., Haussmann, I., Matzku, S., Wenzel, A., Ponta, H., and Herrlich, P. 1991. A new variant of glycoprotein CD44 confers metastatic potential to rat carcinoma cells. Cell 65: 13-24.

Jones, S.M. and Kazlauskas, A. 2001a. Growth factor-dependent signaling and cell cycle progression. FEBS Lett. 490: 110-116.

- 2001b. Growth-factor-dependent mitogenesis requires two distinct phases of signalling. Nat. Cell Biol. 3: 165-172.

Marhaba, R., Bourouba, M., and Zoller, M. 2005. CD44v6 promotes proliferation by persisting activation of MAP kinases. Cell. Signal. 17: 961-973.

Martin, T.A., Harrison, G., Mansel, R.E., and Jiang, W.G. 2003. The role of the CD44/ezrin complex in cancer metastasis. Crit. Rev. Oncol. Hematol. 46: 165-186.

Matter, N., Herrlich, P., and Konig, H. 2002. Signal-dependent regulation of splicing via phosphorylation of Sam68. Nature 420: 691-695.

Muller, W., Schneiders, A., Heider, K.H., Meier, S., Hommel, G., and Gabbert, H.E. 1997. Expression and prognostic value of the CD44 splicing variants v5 and v6 in gastric cancer. J. Pathol. 183: 222-227.

Murphy, L.O., Smith, S., Chen, R.H., Fingar, D.C., and Blenis, J. 2002. Molecular interpretation of ERK signal duration by immediate early gene products. Nat. Cell Biol. 4: 556-564.

Orian-Rousseau, V., Chen, L., Sleeman, J.P., Herrlich, P., and Ponta, H. 2002. CD44 is required for two consecutive steps in HGF/c-Met signaling. Genes \& Dev. 16: 3074-3086.

Ponta, H., Sherman, L., and Herrlich, P.A. 2003. CD44: From adhesion molecules to signalling regulators. Nat. Rev. Mol. Cell Biol. 4: 33-45.

Sherman, L., Wainwright, D., Ponta, H., and Herrlich, P. 1998. A splice variant of CD44 expressed in the apical ectodermal ridge presents fibroblast growth factors to limb mesenchyme and is required for limb outgrowth. Genes \& Dev. 12: 1058-1071.

Sherman, L.S., Rizvi, T.A., Karyala, S., and Ratner, N. 2000. CD44 enhances neuregulin signaling by Schwann cells. J. Cell Biol. 150: 10711084.

Sonobe, S., Miyamoto, H., Nobukawa, B., Izumi, H., Futagawa, T., Ishikawa, N., Yamazaki, A., Uekusa, T., Abe, H., and Suda, K. 2005. 


\section{Cheng et al.}

Prognostic value of CD44 isoform expression in thymic epithelial neoplasms. Cancer 103: 2015-2022.

van der Voort, R., Taher, T.E., Wielenga, V.J., Spaargaren, M., Prevo, R., Smit, L., David, G., Hartmann, G., Gherardi, E., and Pals, S.T. 1999. Heparan sulfate-modified CD44 promotes hepatocyte growth factor/ scatter factor-induced signal transduction through the receptor tyrosine kinase c-Met. J. Biol. Chem. 274: 6499-6506.

von Lintig, F.C., Dreilinger, A.D., Varki, N.M., Wallace, A.M., Casteel, D.E., and Boss, G.R. 2000. Ras activation in human breast cancer. Breast Cancer Res. Treat. 62: 51-62.

Weber, J.D., Raben, D.M., Phillips, P.J., and Baldassare, J.J. 1997. Sustained activation of extracellular-signal-regulated kinase 1 (ERK1) is required for the continued expression of cyclin D1 in G1 phase. Biochem. J. 326: 61-68.

Weg-Remers, S., Ponta, H., Herrlich, P., and Konig, H. 2001. Regulation of alternative pre-mRNA splicing by the ERK MAP-kinase pathway. EMBO J. 20: 4194-4203

Yu, W.H., Woessner Jr., J.F., McNeish, J.D., and Stamenkovic, I. 2002. CD44 anchors the assembly of matrilysin/MMP-7 with heparin-binding epidermal growth factor precursor and ErbB4 and regulates female reproductive organ remodeling. Genes \& Dev. 16: 307-323. 


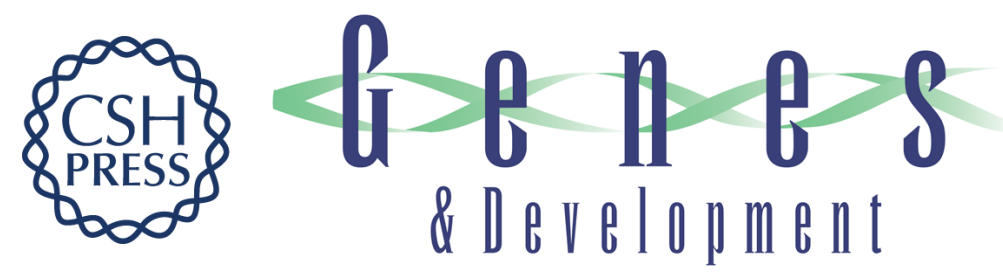

\section{A positive feedback loop couples Ras activation and CD44 alternative splicing}

Chonghui Cheng, Michael B. Yaffe and Phillip A. Sharp

Genes Dev. 2006, 20:

Access the most recent version at doi:10.1101/gad.1430906

\section{Supplemental http://genesdev.cshlp.org/content/suppl/2006/06/09/20.13.1715.DC1 Material}

References This article cites 25 articles, 12 of which can be accessed free at: http://genesdev.cshlp.org/content/20/13/1715.full.html\#ref-list-1

\section{License}

Email Alerting

Receive free email alerts when new articles cite this article - sign up in the box at the top Service

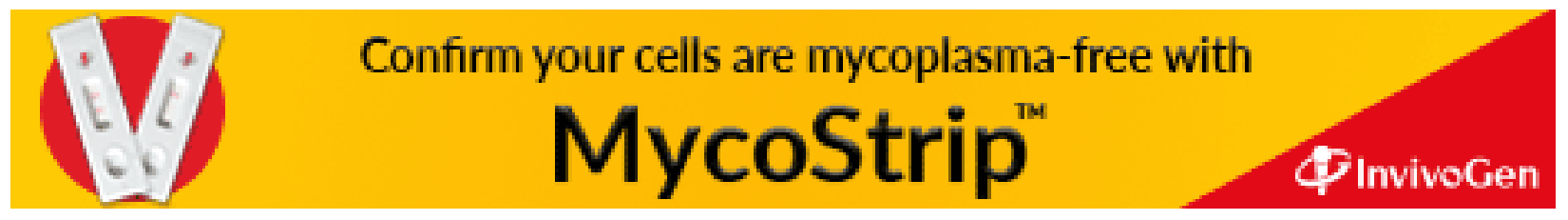

